\title{
A novel $H C F C 1$ variant in male siblings with intellectual disability and microcephaly in the absence of cobalamin disorder
}

\author{
COSTAS KOUFARIS $^{1}$, ANGELOS ALEXANDROU ${ }^{1}$, GEORGE A. TANTELES ${ }^{2}$, \\ VIOLETTA ANASTASIADOU $^{2}$ and CAROLINA SISMANI ${ }^{1}$ \\ Departments of ${ }^{1}$ Cytogenetics and Genomics, and ${ }^{2}$ Clinical Genetics, \\ The Cyprus Institute of Neurology and Genetics, 1683 Nicosia, Cyprus
}

Received October 15, 2015; Accepted December 4, 2015

DOI: $10.3892 /$ br.2015.559

\begin{abstract}
Approximately $10-15 \%$ of intellectual disability (ID) cases are caused by genetic aberrations affecting chromosome X, a condition termed X-linked ID (XLID). Examination by whole-exome sequencing of two male siblings with microcephaly and suspected XLID with an unknown genetic basis revealed that they were both hemizygous for a predicted pathogenic variant (p.Ala897Val) causing a non-synonymous substitution of an evolutionary conserved amino acid within the host cell factor $\mathrm{Cl}$ ( $\mathrm{HCFCl}$ ) gene. Subsequent analysis determined that this was a rare variant not identified in 100 control individuals or in online databases of control individuals. Recent studies have reported mutations affecting HCFCl in patients with ID and dysmorphic features that are associated with defective cobalamin metabolism. Biochemical investigations did not find evidence of an association between the variant identified in the present study and cobalamin metabolic disorder. This study offers further support for mutations of $\mathrm{HCFCl}$ being implicated in XLID and microcephaly, but that these are not necessarily associated with cobalamin disorder.
\end{abstract}

\section{Introduction}

Intellectual disability (ID), defined as the significant impairment of cognitive and adaptive behavior, affects $1.5-2 \%$ of the human population (1), and therefore receives considerable attention from scientists working in the fields of neuroscience and genetics. Studies have shown that $10-15 \%$ of inherited severe ID is the result of genetic aberrations on chromosome X, a condition termed X-Linked ID (XLID) (2). XLID is subdivided into two major categories, syndromic and non-syndromic, based on the presence or absence of specific accompanying clinical, morphological, neurological or

Correspondence to: Dr Carolina Sismani, Department of Cytogenetics and Genomics, The Cyprus Institute of Neurology and Genetics, 6 International Airport Avenue, 1683 Nicosia, Cyprus

E-mail: csismani@cing.ac.cy

Key words: exome, $H C F C 1$, intellectual disability, microcephaly metabolic abnormalities. The borders between non-syndromic and syndromic are not always clearly defined, particularly in small families where few individuals are affected. Small families or individual cases are therefore usually categorized as non-syndromic, contributing to the heterogeneity of this category.

Through the intensive efforts of various research groups and the utilization of powerful new genomic technologies, $>100$ XLID genes have been reported so far, although it is estimated that a substantial fraction of XLID genes remain to be identified $(3,4)$. The recently developed whole-exome sequencing (WES) technology allows the rapid detection of variants located within protein-coding regions of the genome that could not be detected by technologies such as karyotyping and array-comparative genomic hybridization (array-CGH). In the present study, WES was used to screen two brothers with suspected XLID. The analysis identified a novel variant in these patients within $H C F C 1$, a gene recently implicated in XLID.

\section{Materials and methods}

Clinical evaluation of patients. Patient 1 was the first of two male siblings born to healthy non-consanguineous Greek-Cypriot parents and was initially referred to the Clinical Genetics service (The Cyprus Institute of Neurology and Genetics, Nicosia, Cyprus) at the age of 5 years and 2 months for evaluation regarding a history of developmental and speech delay. The patient was born at 39 weeks gestation following a normal vaginal delivery with a birth weight on the 9th percentile, a birth length on the 25-50th percentile, and a birth occipitofrontal circumference (ofc) on the 0.4 th percentile. There were no antenatal concerns, no history of a maternal intercurrent infection or known exposure to teratogens. Developmentally the patient sat without support at the age of 6 months and walked independently at the age of 13 months. The patient presented with moderate developmental motor and speech delay, as well as with severe learning difficulties. Other characteristic findings at the age of clinical evaluation were microcephaly (ofc $<3$ rd percentile) and hyperactivity. The weight and height of the patient were between the 25-50th percentile. The patient had a beaked nose with relatively large ears and synophrys. The hands and feet were normal, including the palmar and plantar creases. 
Patient 2 (the male sibling of patient 1) was referred to the Department of Clinical Genetics at the age of 14 months for evaluation regarding developmental delay. The patient was born prematurely at 35 weeks gestation following an emergency C-section due to fetal distress and prolonged rupture of membranes with a birth weight on the 25 th percentile. At the age of 14 months, the patient was reported to be microcephalic and could only walk with support. On examination at the age of 5 years, the patient had severe developmental and speech delay. The patient was microcephalic (ofc $<3$ th percentile) with myopathic facies, arched eyebrows, an open-mouthed appearance with a Cupid's bow configuration of the upper lip and prognathism. The eyes of the patient were prominent. The patient had a small and narrow alae nasi, triangular nails, bilateral 2-3 toe syndactyly and bilateral pes planus.

The endocrinology and ophthalmology evaluations yielded normal results. Extensive metabolic investigations did not reveal any abnormalities. In both siblings, karyotype, fragile $\mathrm{X}$ syndrome, and high-resolution array-CGH analyses showed normal results.

WES and variant analysis. DNA was extracted from the examined individuals using the Qiagen DNA extraction kit (Qiagen, Hilden, Germany) according to the manufacturer's protocol. WES was performed on DNA samples from the affected male siblings as a service by Oxford Gene Technology (Kidlington, UK). Exome enrichment was performed using the SureSelect exome enrichment kit v1.2 (Agilent Technologies, Thermo Fisher Scientific, Inc., Waltham, MA, USA) and the concentration of each library was determined using the Agilent qPCR NGS Library Quantification kit. Prior to sequencing, the samples were pooled to a concentration of $10 \mathrm{nM}$ per sample. Sequencing of the enriched exome was performed on the Illumina HiSeq 2000 using TruSeq v3 chemistry (Illumina, Inc., San Diego, CA, USA). For the first patient there were 25, 241 and 930 paired reads, $67.48 \%$ of bases were on target, and $92.69 \%$ of target bases were covered at $>20 x$. For the second patient there were 24, 926 and 649 paired reads, $67.39 \%$ of bases were on target, and $92.60 \%$ of bases were covered at $>20 \mathrm{x}$.

For the bionformatics analysis of the WES data the reads were mapped to genome assembly hg19 using the Burrows-Wheeler Aligner package, version 0.6.2 (5). Local realignment of the mapped reads around potential insertion/deletion (indel) sites was carried out with the Genome Analysis Tool kit (GATK) version 1.6 (6). Duplicate reads were marked using Picard version 1.83 (http://picard.sourceforge.net/). Base quality (Phred scale) scores were recalibrated using the covariance recalibration of GATK. SNP and indel variants were named using the GATK Unified Genotyper for each sample. SNP novelty was determined against dbSNP release 135 in February 2013 (7). Further screening of variants was performed using the exome variant server (EVS) database in May 2014 (http://evs.gs.washington.edu/ EVS/). Pathogenicity prediction for identified variants was performed using the Polyphen (http://genetics.bwh.harvard. edu/pph/data/), Sift (http://sift.jcvi.org/), and Condel (http:// bg.upf.edu/fannsdb/) tools.

Sanger sequencing. Purification of polymerase chain reaction products was performed using Exo-Sap ${ }^{\mathrm{IT}}$ (Affymetrix, Santa

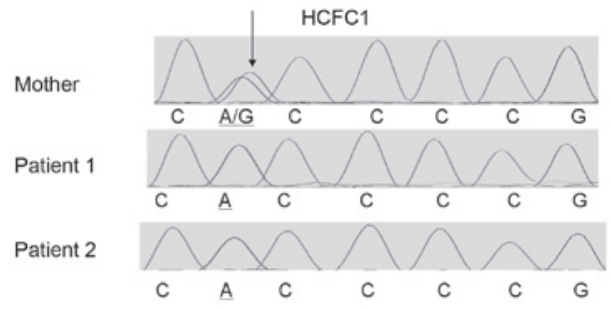

Figure 1. Electropherograms showing the results of Sanger sequencing in the genomic region surrounding rare host cell factor $\mathrm{Cl}$ (HCFCl) variant (ChrX:153221808) in the mother (top lane) and two affected siblings (bottom two lanes). The identified variant is indicated by arrow.

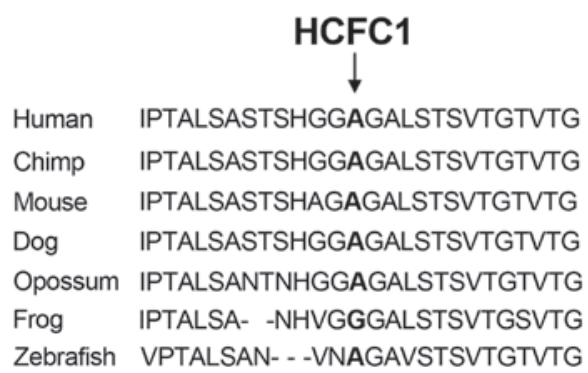

Figure 2. Phylogenetic analysis of host cell factor C1 (HCFC1) protein amino acids sequence in various species. The amino acid affected by the ChrX:153221808 variant is shown in bold and by an arrow.

Clara, CA, USA). Sequencing reactions were performed using Applied Biosystems BigDye terminator v1.1 and products were analyzed on the ABI 3130 sequencer. The Primer sequences are available on request.

\section{Results}

Variations. A total of 46,134 variations were observed for patient 1 and 45,711 variations for patient 2. Of these, there were 45,784 overlapped genes for patient 1 and 45,369 for patient 2. For variants not present in dbSNP 135 there were 3,462 for patient 1 and 3,483 for patient 2. Of these, 675 and 682 , respectively, were predicted to have serious consequences (missense, frameshift, indel, essential splice-site, nonsense and nonstop) respectively. Based on the suspected XLID inheritance of the disease, we prioritized variants detected in the WES data that were: i) Located on the $\mathrm{X}$ chromosome, ii) found in both affected patients, iii) rare variants not present in the dsSNP database, and iv) predicted to have serious phenotypic consequences by in silico tools (Sift, Polyphen or Condel).

Only two variants fulfilled the criteria outlined above: A non-synonymous variant in the HCFC1 gene (ChrX:153,221,808 GRCh37; p.Ala897Val) and a non-synonymous variant in the zinc finger protein, X-linked ( $Z F X)$ gene (ChrX:24,197,706). Further screening of these two variants in the EVS database found that the $\mathrm{HCFCl}$ variant was not present in any of the screened individuals, while the $Z F X$ variant was present in 8 out of 10,555 chromosomes. Additionally, mutations in $Z F X$ had not been previously associated with ID. Consequently, the $Z F X$ variant was less likely to be the cause of the ID in these patients.

Sanger sequencing. The identified $\mathrm{HCFCl}$ variant was present in the two male siblings as confirmed by Sanger sequencing 


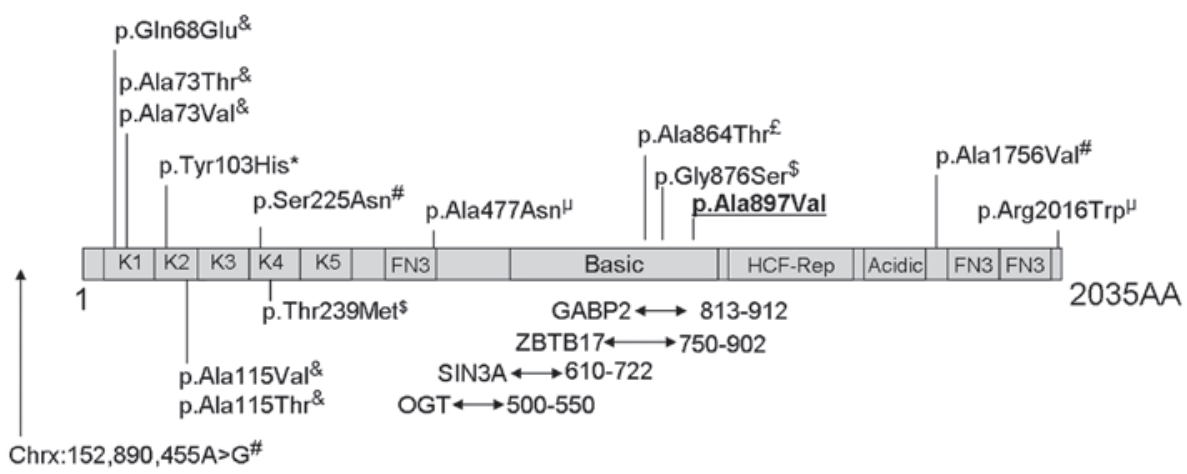

Figure 3. Schematic representation of the host cell factor C1 (HCFC1) protein and its domains based on information from Uniprot (www.uniprot.org; entry P51610) and from published studies. The diagram is not drawn to scale. Kelch domains (K1-K5), fibronectin-type 3 (FN3), basic domain, HCF-proteolysis repeats (HCF-Rep) and acidic domains are shown. Also shown are the protein interaction regions and variants reported in the literature to be associated with intellectual disabilities. Areas of interaction between HCFC1 and the SIN3A, ZBTB17, OGT and GABP2 proteins are indicated by arrows underneath the diagram, with the numbers next to the arrows reporting the location of the interacting domains. The p.Ala897Val variant identified in the present study is underlined in bold. Superscripts above the variants in the diagram indicate the reference reporting this variant: ${ }^{\mathrm{f}}(3),{ }^{\&}(8),{ }^{*}(9),{ }^{\mu}(10),{ }^{\$}(13),{ }^{\sharp}(14)$.

using custom primers and the Big Dye terminator v1.1, while their mother was a heterozygous carrier (Fig. 1). Additionally, Sanger sequencing of the $H C F C l$ variant showed that this was not present in 100 neurotypical individuals from the Cypriot population. This rare variant affects an evolutionary conserved amino acid (Fig. 2), supporting its functional importance.

\section{Discussion}

HCFCl encodes a large protein of $>2,000$ amino acids made up of kelch repeats, HCF-proteolysis repeats, protein interacting domains, basic domain, acidic region, and fibronectin domains (Fig. 3). In recent years studies have reported an association of mutations affecting HCFC1 with ID, metabolic disorders and dysmorphias. Mutations affecting conserved amino acids within the HCFC1 kelch domains have been associated with defective cobalamin metabolism and neurological symptoms by affecting transcription of $M M A H C$ (8). Gérard et al (9) recently reported a p.Tyr103His mutation in two male siblings with ID and cobalamin disorder. These male siblings also exhibited dysmorphic features and microcephaly. Experimental evidence from the examination of patient-derived cell lines and in vitro assays suggest that mutations within kelch domains result in almost complete loss of $\mathrm{HCFC} 1$ function and result in cobalamin disorder, while HCFCl mutations in other regions result in much milder effects on protein function and cobalamin metabolism $(8,10)$. Loss-of-function of the zebrafish homolog of $\mathrm{HCFCl}$ has also been associated with craniofacial abnormalities through dysregulation of $M M A H C$ (11). It has been suggested that mutations in non-kelch domains of $\mathrm{HCFCl}$, which result in partial loss-of-function, cause neurological disorders in the absence of cobalamin deficiency due to HCFC1 having other gene targets that are important for neurological development $(8,10)$.

Examination of patient 1 revealed normal levels of cobalamin, homocysteine and folate in the blood $[436 \mathrm{pg} / \mathrm{ml}$ cobalamin (normal range, 208-964 pg/ml), $9.1 \mathrm{mmol} / 1$ homocysteine (normal range, $5-15 \mathrm{mmol} / \mathrm{l}$ ) and $5.4 \mathrm{ng} / \mathrm{ml}$ folate (normal range, 3-15 ng/ml)]. Consequently, no supporting evidence that the pathogenic phenotype observed in this case is associated with defective cobalamin metabolism was identified.

The variant identified in the present study (p.Ala897Val) is located within the GA-binding protein (GABP) interaction domain. Interaction of GABP with HCFC1 with transcription factors, including GABP, is believed to be essential for the ability of HCFC1 to regulate the transcription of a large number of genes (12). Within the same region, two variants have been identified in an autistic patient (p.Gly876Ser) (13) and in a patient with ID (p.Ala864Thr) (3). The findings of the present study are consistent with mutations within this region of HCFC1 being associated with ID or ASD and with dysmorphic features, without clinical manifestation of cobalamin deficiency.

\section{References}

1. Leonard $\mathrm{H}$ and Wen X: The epidemiology of mental retardation: Challenges and opportunities in the new millennium. Ment Retard Dev Disabil Res Rev 8: 117-134, 2002.

2. De Brouwer AP, Yntema HG, Kleefstra T, Lugtenberg D, Oudakker AR, de Vries BB, van Bokhoven H, Van Esch H, Frints SG, Froyen G, et al: Mutation frequencies of X-linked mental retardation genes in families from the EuroMRX consortium. Hum Mutat 28: 207-208, 2007.

3. Tarpey PS, Smith R, Pleasance E, Whibley A, Edkins S, Hardy C, O'Meara S, Latimer C, Dicks E, Menzies A, et al: A systematic, large-scale resequencing screen of X-chromosome coding exons in mental retardation. Nat Genet 41: 535-543, 2009.

4. Bassani S, Zapata J, Gerosa L, Moretto E, Murru L and Passafaro M: The neurobiology of X-linked intellectual disability. Neuroscientist 19: 541-552, 2013.

5. Li H and Durbin R: Fast and accurate short read alignment with Burrows-Wheeler Transform. Bioinformatics 25: 1754-1760, 2009.

6. McKenna A, Hanna M, Banks E, Sivachenko A, Cibulskis K, Kernytsky A, Garimella K, Altshuler D, Gabriel S, Daly M, DePristo MA: The Genome Analysis Toolkit: A MapReduce framework for analyzing next-generation DNA sequencing data. Genome Res 20: 1297-1303, 20w10.

7. Wheeler DL, Barrett T, Benson DA, et al: Database resources of the National Center for Biotechnology Information. Nucleic Acids Res 35 (Database issue): D5-D12, 2007.

8. Yu HC, Sloan JL, Scharer G, Brebner A, Quintana AM, Achilly NP, Manoli I, Coughlin CR II, Geiger EA, Schneck U, et al: An $\mathrm{X}$-linked cobalamin disorder caused by mutations in transcriptional coregulator HCFC1. Am J Hum Genet 93: 506-514, 2013. 
9. Gérard M, Morin G, Bourillon A, Colson C, Mathieu S, Rabier D, Billette de Villemeur T, Ogier de Baulny $\mathrm{H}$ and Benoist JF: Multiple congenital anomalies in two boys with mutation in HCFC1 and cobalamin disorder. Eur J Med Genet 58: 148-153, 2015.

10. Jolly LA, Nguyen LS, Domingo D, Sun Y, Barry S, Hancarova M, Plevova P, Vlckova M, Havlovicova M, Kalscheuer VM, et al: HCFC1 loss-of-function mutations disrupt neuronal and neural progenitor cells of the developing brain. Hum Mol Genet 24: 3335-3347, 2015.

11. Quintana AM, Geiger EA, Achilly N, Rosenblatt DS, Maclean KN, Stabler SP, Artinger KB, Appel B and Shaikh TH: Hcfclb, a zebrafish ortholog of HCFC1, regulates craniofacial development by modulating mmachc expression. Dev Biol 396: 94-106, 2014.
12. Michaud J, Praz V, James Faresse N, Jnbaptiste CK, Tyagi S, Schütz F and Herr W: HCFC1 is a common component of active human CpG-island promoters and coincides with ZNF143, THAP11, YY1 and GABP transcription factor occupancy. Genome Res 23: 907-916, 2013.

13. Piton A, Gauthier J, Hamdan FF, Lafrenière RG, Yang Y, Henrion E, Laurent S, Noreau A, Thibodeau P, Karemera L, et al: Systematic resequencing of $\mathrm{X}$-chromosome synaptic genes in autism spectrum disorder and schizophrenia. Mol Psychiatry 16: 867-880, 2011.

14. Huang L, Jolly LA, Willis-Owen S, Gardner A, Kumar R, Douglas E, Shoubridge C, Wieczorek D, Tzschach A, Cohen M, Hackett A, Field M, Froyen G, Hu H, Haas SA, Ropers HH, Kalscheuer VM, Corbett MA and Gecz J: A noncoding, regulatory mutation implicates $\mathrm{HCFC} 1$ in nonsyndromic intellectual disability. Am J Hum Genet 91: 694-702, 2012. 